\title{
A noção de derivada parcial: níveis de conhecimento esperados dos estudantes em função das organizações matemáticas e didáticas prescritas
}

\author{
The notion of partial derivative: expected knowledge levels of students according \\ to prescribed mathematical and didactical organizations
}

\author{
Marlene Alves Dias \\ alvesdias@ig.com.br \\ Pedro Mateus \\ pmateuszulu@gmail.com \\ Francisco Regis Vieira Alves \\ fregis@ifce.edu.br
}

\begin{abstract}
Resumo
Iniciamos este trabalho apresentando nosso objetivo que é compreender as dificuldades apresentadas pelos estudantes no processo de ensino e aprendizagem da noção de derivada parcial. Para tal, consideramos como quadro teórico central a abordagem teórica em termos dos três níveis de conhecimento esperados dos estudantes definida por Robert. Apoiamo-nos ainda nas noções de quadro, segundo definição de Douady e na noção de ponto de vista apresentada por Rogalski, além de elementos da Teoria Antropológica do Didático de Chevallard e seus colaboradores. Metodologicamente, consideramos o método da pesquisa documental, que foi desenvolvido por meio de uma grade de análise norteada pelo modelo de Dias. As análises permitem avançar algumas considerações sobre os conhecimentos prévios necessários para a aprendizagem da noção de derivada parcial e suas aplicações, destacando a importância das aplicações em outras ciências que necessitam de atenção específica, uma vez que os conhecimentos associados a estas ciências não são desenvolvidos na educação básica.
\end{abstract}

Palavras Chave: Níveis de conhecimento; Derivada parcial; Teoria Antropológica do Didático.

\begin{abstract}
We would like to start by presenting our objective, which is to understand the difficulties presented by students in the teaching-learning process of the notion of partial derivative. To do so, we consider as central the theoretical approach defined by Robert, related to the three levels of expected knowledge from the students. We are also supported by the notions of setting according to Douady's definition and Rogalski's point of view, as well as by the elements of the anthropological theory of didactics according to Chevallard and his collaborators. Methodologically, we have used documentary research developed through a grid of analysis according to Dias's model. The analyzes allow us to advance some considerations on the necessary previous knowledge to learn the notion of partial derivative and its applications, highlighting the importance of the applications in other sciences that need specific attention, since the knowledge associated to these sciences are not developed in the basic education.
\end{abstract}

Keywords: Levels of knowledge; Partial derivative; Anthropological Theory of Didactics.

\section{Introdução}

Este estudo é parte de uma pesquisa sobre como a abordagem teórica em termos dos três níveis de conhecimento esperados dos estudantes de Robert (1998) pode auxiliar a compreender as dificuldades apresentadas pelos estudantes no processo de ensino e aprendizagem de Matemática, em particular, de cálculo diferencial e integral. 
Desta forma, nossa proposta foi analisar as possíveis dificuldades relativas aos conhecimentos prévios de Matemática necessários, tanto do ponto de vista do professor, como do estudante, quando se introduz a noção de derivada parcial, ou seja, qual parte fica a cargo do professor e qual parte compete ao estudante.

Sendo assim, fizemos um estudo detalhado do ensino da noção de derivada parcial para compreender melhor como trabalhar esta noção nos diferentes cursos do Ensino Superior, visando desenvolver uma flexibilidade cognitiva, que permita ao aluno ser capaz de resolver problemas, tanto em nível mobilizável como disponível.

Observamos aqui que a noção de flexibilidade cognitiva, conforme Dias (1998), corresponde à capacidade de trabalhar em diversos quadros, podendo-se mudar de quadro quando necessário, sem que para isso seja feito qualquer apelo explícito; a utilizar a representação mais adequada para a resolução de um problema, articular diferentes pontos de vista e concepções; saber planejar, analisar, desenvolver, controlar e justificar o trabalho matemático, isto é, ser capaz de trabalhar no nível disponível sempre que necessário, articulando os diferentes conhecimentos em jogo nas tarefas.

No referencial teórico, apresentamos as noções de níveis de conhecimento esperados dos estudantes, quadro, mudança de quadros, pontos de vista e ainda a definição de objetos ostensivos e não ostensivos que correspondem aos objetos de manipulação e evocação do trabalho matemático, introduzidos por Chevallard (1994), respectivamente, como os ingredientes que possibilitam o desenvolvimento das organizações matemáticas ou praxeologias matemáticas que também estão definidas no referencial teórico.

Considerando que não se pode trabalhar uma determinada noção da mesma forma, não importando qual o contexto em que se encontra, observamos que os três níveis de conhecimento esperados dos estudantes nos auxiliam a estabelecer as praxeologias que precisam ser trabalhadas e aprofundadas para desenvolver os níveis mobilizável ou disponível, o que equivale a aplicar com autonomia e flexibilidade os conhecimentos matemáticos desenvolvidos nas diferentes etapas escolares.

Assim, fomos conduzidos a estudar as condições necessárias para que o estudante possa articular de forma autônoma e flexível tarefas relacionadas à noção de derivada parcial.

Para isso, a pesquisa foi orientada pelo seguinte questionamento:

1) Quais as relações institucionais existentes?

2) Quais os conhecimentos matemáticos necessários para compreender a noção de derivada parcial no Ensino Superior e poder aplicá-la de forma eficaz, quando necessário?

3) Sobre quais níveis de conhecimento essas necessidades se manifestam: técnicos, mobilizáveis e disponíveis, quando se deseja atingir uma aprendizagem com significado? 
Para abordar as questões acima, analisamos o funcionamento institucional dos três níveis de conhecimento para a noção de derivada parcial. Essa análise institucional é feita por meio da pesquisa de um conjunto de dez livros didáticos com proposta para o Ensino Superior de Matemática e outras ciências, isto é, considerando os livros analisados como as relações institucionais existentes, uma vez que os mesmos, em geral, são indicados na bibliográfica básica e na complementar de diversos cursos de ciências exatas do Ensino Superior.

Com esta análise, fizemos um estudo detalhado do ensino dessa noção, para compreender melhor como introduzi-la de modo a desenvolver um trabalho centrado em um tratamento que possibilite ao estudante trabalhar de forma flexível.

Escolhemos assim, como referencial teórico central dessa pesquisa, a abordagem em termos dos três níveis de conhecimento esperados dos estudantes, segundo definição de Robert (1998), pois esta abordagem propicia compreender quais conhecimentos privilegiar e quais as outras noções em jogo de que os estudantes devem dispor, o que pode auxiliar a desenvolver diagnósticos específicos para o início de trabalho com diferentes grupos de estudantes, levando em conta a contextualização dos saberes para cada grupo.

Para o desenvolvimento de nossas análises, respaldamo-nos na abordagem teórica em termos de quadros e mudança de quadros à luz da definição de Douady $(1984,1992)$ e em alguns elementos da Teoria Antropológica do Didático (TAD) de Chevallard e seus colaboradores.

Apresentamos a seguir o referencial teórico da pesquisa, iniciando com uma breve descrição da abordagem teórica em termos dos três níveis de conhecimento esperados dos estudantes.

\section{Referencial teórico da pesquisa}

Observamos que nossa proposta é compreender como auxiliar os estudantes a utilizarem as noções matemáticas associadas à noção de derivada parcial de forma mobilizável ou disponível.

Consideramos, mais particularmente, a abordagem em termos dos três níveis de conhecimento esperados dos estudantes, consoante definição de Robert (1998), por tratar-se de uma análise mais global, pois, após identificar praxeologias matemáticas, quadros, pontos de vista, ostensivos e não ostensivos, podemos optar por aquelas que nos permitem desenvolver os níveis mobilizável e disponível em relação à noção de derivada parcial, assim como mostrar quais os níveis de conhecimento esperados em relação aos outros 
conhecimentos que estão em jogo nas praxeologias matemáticas que lhes são propostas quando da introdução desta nova noção.

A seguir apresentamos brevemente as noções de nível de conceituação e nível de conhecimento esperado dos estudantes, de acordo com definições de Robert (1997, 1998).

\section{Os três níveis de conhecimento esperados dos estudantes}

Iniciamos considerando a noção de níveis de conceituação, observando que esta é importante, quando desejamos desenvolver um estudo sobre os níveis de conhecimento esperados dos estudantes, uma vez que estes são relativos, ou seja, dependem do nível de conceituação em que nos encontramos.

Desse modo, segundo Robert (1997), os níveis de conceituação são os marcos que podemos identificar ao longo do ensino das noções de determinado campo conceitual. Muitas noções matemáticas podem ser abordadas em vários níveis de conceituação, sempre parcialmente encaixados, pois os objetos iniciais mudam, eles se tornam mais gerais, o que possibilita introduzir novas estruturas, mais ricas, e para isso necessitam de um novo formalismo, a eles adaptado. Em geral, os exercícios ditos teóricos de um determinado nível correspondem aos teoremas do nível seguinte. Dessa forma, várias ordens de apresentação são sempre possíveis, não existe hierarquia absoluta entre esses níveis, que, pelo menos durante os estudos, dependem apenas do ensino efetivo, ou seja, das diferentes propostas de ensino.

Após considerar que o ensino das noções matemáticas associadas a um campo conceitual depende da escolha da ordem de apresentação, Robert (1998) define os três níveis de conhecimento esperados dos estudantes, a saber:

O nível técnico corresponde a um trabalho isolado, local e concreto. Está relacionado principalmente às ferramentas e definições utilizadas em uma determinada tarefa.

Para a noção de derivada parcial, pode-se considerar o seguinte exemplo:

- $\quad$ Considere a função $z=f(x, y)$ dada por $z=x^{2}+3 x y+y^{2}-3 x-3 y$. Calcule $\frac{\partial z}{\partial x}$.

O nível mobilizável corresponde a um início de justaposição de saberes de determinado quadro, podendo até corresponder a uma organização. Vários métodos podem ser mobilizados. O caráter ferramenta e objeto do conceito estão em jogo, mas o que se questiona é explicitamente pedido. Se um saber é identificado, ele é considerado mobilizado se ele é acessível, isto é, se o estudante o utiliza corretamente. 


\section{Exemplo:}

- $\quad$ Seja $\mathrm{g}(\mathrm{x}, \mathrm{y})=\phi(\mathrm{x} / \mathrm{y})$ a função $\phi: \mathbf{R} \rightarrow \mathrm{R}$ uma função de uma variável real, diferenciável e tal que $\phi^{\prime}(\mathrm{x} / \mathrm{y})=4$. Verifique que $\mathrm{x} \frac{\partial g}{\partial x}(\mathrm{x}, \mathrm{y})+\mathrm{y} \frac{\partial g}{\partial y}(\mathrm{x}, \mathrm{y})=0$ para todo $(\mathrm{x}, \mathrm{y})$ $\in \mathrm{IR}^{2}$, com y $\neq 0$. (GUIDORIZZI, 2004, p. 182). Para resolver esta questão, o estudante deve dispor de conhecimentos sobre função composta e derivada da função composta, que em relação à x será dada por $\frac{\partial g}{\partial x}(\mathrm{x}, \mathrm{y})=\phi^{\prime}(\mathrm{x} / \mathrm{y}) \cdot \frac{\partial}{\partial x}(\mathrm{x} / \mathrm{y})$. Apesar de estar explícito no enunciado que se trata de derivada parcial, o estudante precisa mobilizar seus conhecimentos sobre como determinar esta derivada para a função dada.

O nível disponível corresponde a saber responder corretamente o que é proposto sem indicações, a poder, por exemplo, dar contraexemplos (encontrar ou criar), mudar de quadro (fazer relações), aplicar métodos não previstos.

Esse nível de conhecimento está associado à familiaridade, ao conhecimento de situações de referência variadas que o estudante sabe que conhece (servem de terreno de experimentação), ao fato de dispor de referências, de questionamentos, de uma organização. Pode funcionar para um único problema ou possibilitando fazer resumos.

Exemplo:

- Estudar com relação a máximos e mínimos locais a função $f(x, y)=x^{4}+y^{4}+4 x$ + 4y. (GUIDORIZZI, 2004, p. 315) Neste caso, o estudante deve dispor de conhecimentos sobre a determinação de máximos e mínimos locais, que supõem o cálculo das derivadas parciais de primeira ordem que serão igualadas a zero para se determinar os pontos críticos. Essa operação exige conhecimentos sobre as noções de sistemas de equações lineares e não lineares e raízes de polinômios para a determinação destes pontos. Para o estudo dos máximos e mínimos, os estudantes devem dispor ainda de conhecimentos sobre como determinar as derivadas parciais de segunda ordem e do hessiano para utilizar o critério de análise e verificar a existência de máximos e mínimos locais. Observa-se ainda que, para o cálculo da segunda derivada, dependendo da função, os estudantes devem dispor de conhecimentos sobre fatoração, que permitem simplificar a primeira derivada antes de se lançarem no cálculo da segunda derivada. Ao determinar os valores de x e y para os pontos críticos, é preciso lembrar-se da necessidade de combinar os diferentes valores encontrados para determinar estes pontos. Nesses casos, verifica-se a necessidade de planejamento e de controle do trabalho efetuado para justificar a resposta encontrada. 
$\mathrm{Na}$ sequência, apresentamos as noções de quadro e mudança de quadros, segundo definição de Douady $(1984,1992)$.

\section{As noções de quadro e mudança de quadros conforme definição de Douady}

As noções de quadro e mudança de quadros foram introduzidas por Douady (1984, 1992) que, a partir de uma análise epistemológica sobre o trabalho do matemático profissional, coloca em evidência a dualidade dos conceitos matemáticos, os quais, em geral, funcionam como ferramentas implícitas e, em seguida, explícitas da atividade matemática antes de adquirirem o status de objeto e de serem trabalhados como tal. Seguindo esta perspectiva, a pesquisadora considera ainda o papel desempenhado pelas mudanças de quadros nas atividades e na produção matemática e as transpõe para o ensino por meio dos jogos de quadros.

Ressaltamos que em nossas análises identificamos os quadros: algébrico, geométrico e analítico, sendo este último constituído dos objetos do Cálculo Diferencial e Integral.

Conforme Douady $(1984,1992)$, uma ferramenta implícita corresponde a um conceito em elaboração, podendo durar vários anos. Já uma ferramenta explícita está associada a um conceito ou a uma noção utilizada intencionalmente para resolver um problema e um objeto é definido como um componente cultural que ocupa um lugar bem determinado no complexo edifício do saber matemático, sendo reconhecido socialmente. Como exemplo, podemos citar a noção de fatoração algébrica que, na disciplina de Cálculo Diferencial e Integral, precisa funcionar como ferramenta explícita, quando consideramos o quadro algébrico.

O objeto matemático é parte de um edifício mais amplo que é o saber matemático, constituindo assim o que ela denomina quadro, que corresponde a um ramo da Matemática, das relações entre os objetos, de suas formulações eventualmente diversas e das imagens mentais que lhes são associadas. As imagens mentais são essenciais, pois funcionam como ferramentas dos objetos do quadro. Dois quadros podem conter os mesmos objetos, mas diferirem pelas imagens mentais e problemáticas desenvolvidas.

Douady $(1984,1992)$ define as mudanças de quadros como meios para se obterem formulações diferentes de um problema, que podem ou não ser equivalentes, mas que possibilitam um novo acesso às dificuldades encontradas e permitem utilizar novas ferramentas e técnicas que não eram adequadas para a formulação inicial. As traduções de um quadro em outro terminam sempre em resultados desconhecidos, em novas técnicas, favorecendo assim a criação de novos objetos matemáticos, enriquecendo, tanto o quadro original, como os quadros auxiliares de trabalho. 
A seguir apresentamos a noção de ponto de vista que é menos precisa que a noção de quadro, mas que auxilia a compreender melhor esta noção e identificar outras formas de observar os objetos matemáticos.

\section{A noção de ponto de vista}

Segundo Rogalski (2001), dois pontos de vista diferentes sobre um objeto matemático são diferentes maneiras de observá-los, de fazê-los funcionar, eventualmente de defini-los.

Nesse sentido, observar um objeto em diferentes quadros é considerar diferentes pontos de vista. Mas podem-se considerar vários pontos de vista em um mesmo quadro.

Um exemplo interessante de pontos de vista sobre o objeto matemático derivada é o de Thruston (1994), que apresenta uma lista não exaustiva das diferentes maneiras com que os matemáticos são capazes de conceber a noção de derivada de uma função, como podemos notar na citação a seguir.

[...] 1. Infinitesimal: a razão da mudança infinitesimal do valor da função pela mudança infinitesimal da variável. 2. Simbólico: a derivada de $\mathrm{x}^{\mathrm{n}}$ é $\mathrm{nx}^{\mathrm{n}-1}$, a derivada se $\operatorname{sen}(\mathrm{x})$ é $\cos (\mathrm{x})$, a derivada de fog é f'og*', etc. 3. Lógico: $\mathrm{f}(\mathrm{x})=\mathrm{d}$ se e somente se, para cada $\varepsilon$, existe um $\delta$ tal que se $0<|\Delta x|<\delta$, então $\left|\frac{f(x+\Delta x)-f(x)}{\Delta x}-d\right|<\varepsilon$. 4. Geométrico: a derivada é a inclinação da tangente ao gráfico, se o gráfico tem uma tangente nesse ponto. 5. Taxa: a velocidade instantânea de $\mathrm{f}(\mathrm{t})$ se t é o tempo. 6. Aproximação: a derivada de uma função é a melhor aproximação linear dessa função próxima do ponto. 7. Microscópico: a derivada de uma função é o limite do que se observa com um microscópio aumentando cada vez mais (THURSTON, 1994, p. 5).

A seguir, apresentamos os elementos da Teoria Antropológica do Didático que utilizamos na pesquisa, mostrando seu interesse para a análise dos três níveis de conhecimento esperados dos estudantes.

\section{Os três níveis de conhecimento esperados dos estudantes e a teoria antropológica do didático (TAD)}

Apresentamos aqui apenas alguns elementos da TAD que nos auxiliaram no desenvolvimento da pesquisa, a saber: as noções de relação institucional e pessoal que são definidas em Chevallard (1992, 1998, 2015) a partir das noções fundamentais de objeto, pessoa e instituições. Assim, na perspectiva antropológica, a primeira noção fundamental é a de objeto, que corresponde a toda entidade, material ou imaterial, que existe para pelo menos um indivíduo. Desse modo, a noção de objeto é a mais geral, pois, para o autor, tudo é objeto, inclusive as pessoas. 
Observamos ainda a importância de ponderar sobre a noção de obra $O$ que, segundo Chevallard (1998), também corresponde a um objeto, uma vez que na teoria, as obras são consideradas como qualquer parte de um complexo de organizações praxeológicas.

Conforme o pesquisador, a segunda noção fundamental é a de relação pessoal de um indivíduo $x$ com um objeto $o$, que corresponde à expressão pela qual designamos o sistema, representado por $R(x, o)$, de todas as interações, sem exceção, que uma pessoa $x$ pode ter com o objeto $o$, isto é, $x$ pode manipulá-lo, utilizá-lo, falar sobre ele, sonhar com ele etc. $\mathrm{O}$ pesquisador explicita que ter uma relação pessoal não vazia com o objeto $o$ pode significar simplesmente ter ouvido falar de $o$, indicando assim que em TAD o verbo conhecer pode designar uma ampla variedade de relações.

A terceira noção fundamental é a de pessoa, que é definida pelo par formado por um indivíduo $x$ e o sistema de relações pessoais $R(x, o)$ num dado momento da história de $x$. Dessa forma, quando um objeto $o$ existe para uma pessoa $x$, ou seja, quando $R(x, o) \neq \phi$, dizemos que x conhece $o$ e que a relação $R(x, o)$ indica a maneira que $x$ conhece $o$.

A quarta noção fundamental é a de instituição que, para o autor, são obras de um tipo particular que proporcionam e impõem a seus sujeitos maneiras próprias de fazer, ou seja, praxeologias bem determinadas. Assim, por exemplo, um livro didático é uma obra e a classe que possibilita a utilização desse livro é uma instituição.

Desse modo, a "teoria do conhecimento" esboçada para os indivíduos é transferida para as instituições, ou seja, dado um objeto $o$, uma instituição I e uma posição $p$ em $I$, denominamos relação institucional a $o$ em posição $p$, e indicamos $R_{I}(p, o)$, a relação com o objeto $o$ que deveria ser, idealmente, aquela dos sujeitos de $I$ em posição $p$. Dizer que $x$ é um bom sujeito de $I$ em posição $p$ é o mesmo que afirmar que a relação pessoal do indivíduo $x$ está em conformidade ou é adequada à relação institucional em posição $p$, que indicamos $R(x$, $o) \cong R_{I}(p, o)$. Para diversos objetos $o$, temos $R_{I}(p, o)=\phi$, isto é, os sujeitos de $I$ em posição $p$ não são conduzidos a conhecer o objeto $o$.

$\mathrm{O}$ autor define ainda universo cognitivo pessoal de $x$ ao objeto $o$ e indica por $\operatorname{Co}(x)$ o conjunto de todas as relações pessoais não vazias de um indivíduo $x$ com um objeto $o$, ou seja, $\mathrm{Co}(x)=\{\mathrm{R}(x, o) / \mathrm{R}(x, o) \neq \phi\}$.

Ainda, de acordo com Chevallard (1998), ao se tornar sujeito de $I$ em posição $p$, um indivíduo $x$, que é sempre uma pessoa, submete-se às relações institucionais $R_{I}(p, o)$, que irão (re)modelar, (re)formar suas relações pessoais, de modo que, se $o$ existe para os sujeitos que estão na posição $p$, a relação pessoal de $x$ a $o, R(x, o)$ tende a ser semelhante à relação 
institucional $R_{I}(p, o)$, a menos que x se revele ser um mau sujeito de $I$. De forma geral, nossas relações pessoais são fruto de nossa história de sujeições institucionais passadas e presentes.

Ressaltamos aqui que, segundo Chevallard (2015), as noções acima apresentadas precisam ser parametrizadas pelo tempo.

Sendo assim, consideramos importante articular a noção de níveis de conhecimento esperados dos estudantes com a de relações pessoais com um objeto $o$, lembrando que esta depende das relações institucionais a que os mesmos se submeteram.

Como para compreender as relações institucionais é preciso identificar as organizações matemáticas e didáticas desenvolvidas nas instituições, apresentamos a seguir a noção de praxeologia.

Segundo Bosch e Chevallard (1999), a noção de organização praxeológica ou praxeologia corresponde aos tipos de tarefas $(T)$ que, para serem executadas, necessitam de uma maneira de fazer que os autores denominam técnica $(\tau)$. A associação tarefa-técnica é definida como um saber fazer que não sobrevive isoladamente, solicitando um ambiente tecnológico-teórico, que equivale a um saber formado por uma tecnologia ou discurso tecnológico $(\theta)$, ou seja, um discurso racional que justifica e torna a técnica compreensível, e de uma teoria $(\Theta)$ que justifica e esclarece a tecnologia utilizada.

O sistema composto por tipo de tarefa, técnica, tecnologia e teoria $[T, \tau, \theta, \Theta]$ constitui o que os autores denominam organização praxeológica ou praxeologia, sendo ela que articula uma parte prático-técnica, que corresponde ao saber fazer, a uma parte tecnológica teórica, que equivale ao saber.

A noção de organização didática é definida por Chevallard (1998) como a reconstrução ou a transposição da organização matemática na sala de aula.

Considerando que tanto a organização matemática como a organização didática são tarefas a serem realizadas pelo professor, parece-nos importante que este possa identificar claramente os níveis de conhecimentos esperados dos estudantes nas praxeologias a serem desenvolvidas.

Observamos aqui que estas praxeologias podem variar segundo o nível de conceituação em que o grupo de estudantes se encontra, ficando a cargo do professor escolher tarefas cujo nível de conhecimento seja o mais adequado à sua turma.

Consideramos ainda as noções de objetos ostensivos e não ostensivos, uma vez que são elas que permitem manipular as técnicas e evocar os conhecimentos utilizados nessa manipulação. 
Ressaltamos que após considerar que em toda atividade humana somos chamados a realizar diferentes tipos de tarefas e que para cada uma delas existe uma técnica, Chevallard (1994) apresenta as seguintes questões: De que é feita uma técnica? De que ingredientes se compõe? Em que consiste a "execução" de uma técnica?

Para respondê-las, o autor distingue dois tipos de objetos: os ostensivos e os não ostensivos. Os primeiros são objetos que têm para nós uma forma material, sensível. Exemplos: objetos materiais (caneta, compasso etc.); gestos (ostensivos gestuais); palavras, e mais genericamente o discurso (ostensivos discursivos); esquemas, desenhos, grafismos (ostensivos gráficos); escritas e formalismos (ostensivos escriturais).

A característica dos ostensivos é que eles podem ser manipulados, não só no sentido tátil estrito (como um compasso, uma caneta etc.), mas também em sentido amplo (pela voz, pelo olhar etc.). Ao contrário, os objetos não ostensivos, que denominamos usualmente noções, conceitos, ideias etc., não podem ser manipulados, mas só evocados por manipulação dos ostensivos associados. Chevallard (1994) observa existir uma dialética necessária entre ostensivos e não ostensivos, pois os primeiros são manipulados por meio de regras, cuja distinção é feita pelos não ostensivos, enquanto estes últimos são evocados por meio da manipulação dos ostensivos.

Tanto Robert como Chevallard consideram a importância da linguagem não somente como uma forma de comunicação, mas como um processo que conduz a exigências suplementares (planejar, desenvolver, justificar e controlar o trabalho desenvolvido), tanto da parte dos professores como dos estudantes, o que se explicita na teoria de Chevallard por meio das noções de objetos ostensivos e não ostensivos e que é considerado, na abordagem teórica de Robert, por meio das noções de quadros e mudança de quadros, pontos de vista e registro de representação semiótica, conforme definição de Duval (1995), mas que não é explicitado nesta pesquisa, uma vez que utilizamos os ostensivos, que, conforme Bosch e Chevallard (1999), têm uma valência semiótica (os sinais) e uma valência instrumental que corresponde à capacidade de integrar-se nas manipulações técnicas, tecnológicas e teóricas.

A partir do referencial teórico acima considerado, optamos pela metodologia da pesquisa descrita na sequência.

\section{Metodologia da pesquisa}

O estudo proposto no presente trabalho efetua-se em duas fases:

Na primeira, estudam-se as propostas institucionais para o desenvolvimento da noção de derivada parcial nos cursos de Ciências Exatas na tentativa de melhor compreender os níveis de conhecimento necessários para alcançar os objetivos do estudo desta noção nos 
cursos de Cálculo Diferencial e Integral no Ensino Superior, em particular, nos cursos de Licenciatura em Matemática.

$\mathrm{Na}$ segunda fase, elabora-se uma grade de análise que viabiliza verificar os níveis privilegiados para a abordagem da noção de derivada parcial no Ensino Superior. Essa grade é aplicada nos livros didáticos com o objetivo de observar as regularidades e diferenças institucionais existentes nas propostas. Neste trabalho, expomos apenas os resultados das análises de três livros didáticos, dois deles escolhidos por apresentarem abordagens que diferem entre si em relação à proposta de tipos de tarefas intra e extramatemáticas e o terceiro por ser o mais indicado nas bibliografias básicas dos cursos de Matemática e outras Ciências. O resultado da análise dos livros foi apresentado respeitando o critério de escolha.

Este estudo permite estabelecer os diferentes tipos de tarefas associadas à noção de derivada parcial que intervêm nos cursos de Matemática e outras Ciências para os estudantes do Ensino Superior.

\section{A grade de análise}

O propósito dessa grade é servir como um instrumento que torne possível analisar os diferentes níveis de conhecimento exigidos dos estudantes num curso de introdução ao cálculo, quando se trabalha com a noção de derivada parcial.

Essa grade possibilita analisar os três níveis de conhecimento (técnico, mobilizável e disponível) exigidos dos estudantes:

Em função dos tipos de tarefas associadas à noção de derivada parcial encontradas em um curso de introdução ao cálculo;

- $\quad$ Em função das variáveis dessas tarefas, para as quais será dada ênfase ao nível de conhecimento pedido explicitamente no enunciado e aos diferentes níveis de conhecimento de outras noções que devem ser utilizados para a solução da tarefa.

Primeiramente, estudam-se as diferentes tarefas usualmente encontradas para a introdução da noção de derivada parcial e, em seguida, quais os diferentes níveis de conhecimento exigidos dos estudantes na solução dessas tarefas. Para especificar a tarefa em relação aos diferentes níveis de conhecimento exigidos, consideram-se as variáveis das tarefas definidas abaixo:

- Nível de conhecimento exigido pela tarefa;

- Ostensivos dados no enunciado;

- Quadro em que a tarefa é enunciada;

- Ostensivos necessários para a solução da tarefa;

- Níveis de conhecimento necessários para a execução da tarefa em relação às noções que serão utilizadas. 


\section{Exemplo de funcionamento da grade em uma tarefa}

Propondo-se o tipo de tarefa: Resolver uma situação contextualizada, utilizando derivadas parciais. Por exemplo: Deseja-se construir uma caixa, sem tampa, com a forma de um paralelepípedo - retângulo e com $1 \mathrm{~m}^{3}$ de volume. O material a ser utilizado nas laterais custa o triplo do que será utilizado no fundo. Determine as dimensões da caixa que minimizam o custo do material.

Nesta tarefa particular, as variáveis são as seguintes:

Nível de conhecimento exigido pela tarefa: disponível.

Ostensivos dados no enunciado: língua natural, geométrico e funcional.

Quadro em que a tarefa é enunciada: geometria espacial.

Ostensivos necessários para a solução da tarefa: Para executar a tarefa, os estudantes devem passar dos ostensivos de língua natural para o ostensivo geométrico de representação da caixa e ostensivo simbólico funcional de representação do volume da caixa que irão auxiliar na conversão do enunciado do ostensivo de língua natural para o ostensivo funcional por meio do objeto matemático função de duas variáveis reais a valores reais.

Níveis de conhecimento necessários para a execução da tarefa em relação às noções que serão utilizadas: Para responder à questão, o estudante deverá dispor de conhecimentos sobre: conversão de uma tarefa enunciada por meio do ostensivo em língua natural para o ostensivo simbólico funcional, a noção de volume de um paralelepípedo retângulo e a noção de área de um retângulo para encontrar a função volume. Em relação à noção de derivada parcial, o estudante deve dispor de conhecimentos de derivadas parciais de primeira e segunda ordem, de hessiano e das condições para que um ponto crítico seja máximo ou mínimo local e global. Para o cálculo dos pontos críticos, o estudante deve dispor de um método de resolução de sistemas não lineares e de meios para controlar e justificar os resultados encontrados.

Esse exemplo mostra por que o professor, ao escolher uma tarefa deste tipo, deve levar em conta os conhecimentos prévios dos estudantes ou pelo menos estar preparado para revisitar os pontos que, mesmo supostos disponíveis, ainda apresentam dificuldades. Ao considerar os níveis de conhecimento necessários para a execução da tarefa, apresentamos implicitamente as técnicas, tecnologias e teorias que correspondem à praxeologia deste tipo de tarefa.

Na sequência, apresentamos uma breve análise dos resultados encontrados para os três livros analisados dentre onze.

\section{Resultados da análise dos três livros didáticos}

Apresentamos no presente trabalho os resultados de três entre os dez livros analisados, pois esses tendem a seguir um mesmo modelo, isto é, após introduzir as noções de funções de duas ou mais variáveis, limite e continuidade, consideram a noção de derivada parcial, funções diferenciáveis e estudo dos máximos e mínimos. Em todos os livros analisados, 
verificamos que existe uma preocupação em trabalhar os três níveis de conhecimento, sendo o nível técnico o que aparece em primeiro plano.

Desta forma, a análise foi estruturada em torno das seguintes questões:

$\square$ Como é introduzida a noção de derivada parcial, quais representações são utilizadas e como elas se articulam?

$\square$ Que pesos respectivos ocupam os níveis: técnico, disponível e mobilizável nas tarefas propostas aos estudantes?

$\mathrm{Na}$ sequência, apresentamos os resultados da análise de três livros didáticos que diferem entre si, principalmente em função do privilégio dado ao tratamento intra e extra matemático.

\section{Análise da obra: Um Curso de Cálculo de Guidorizzi (2004)}

Após introduzir as noções de funções de diversas variáveis reais a valores reais e o estudo do domínio e imagem destas funções, isto é, seu tratamento por meio do ostensivo algébrico e do ostensivo gráfico da curva que representa este domínio, o que permite considerar as curvas de nível e suas representações gráficas no caso das funções de duas variáveis reais. $\mathrm{O}$ autor segue propondo o estudo de limite e continuidade destas funções e introduz a noção de derivada parcial utilizando apenas o ostensivo algébrico para as tarefas propostas aos estudantes, o que conduz a proposição de um grande número de tarefas nas quais os níveis de conhecimento privilegiados são o nível técnico e mobilizável. Para as tarefas que exigem o nível disponível, tanto em relação a conhecimentos prévios da própria matemática ou de outras ciências, o autor, em geral, indica por meio de pistas no enunciado os caminhos a seguir.

Observamos que as tarefas propostas nos livros didáticos estão, em geral, no final do capítulo, sempre associadas à noção que se está trabalhando, o que já é uma indicação da noção a ser utilizada, o que permite considerá-la no nível mobilizável em relação à noção de derivada parcial para este nosso estudo.

Trata-se de uma obra mais indicada aos estudantes do curso de Matemática na qual o autor tem o cuidado de trabalhar exemplos de outros domínios da própria matemática o que auxilia a articulação de quadros e pontos de vista.

\section{Análise da Obra Cálculo: Um Curso Moderno e suas Aplicações de Hoffmann et Bradley (2002)}

Assim como o livro de Guidorizzi (2004) a obra de Hoffmann et Bradley (2002) apresenta as noções de funções de diversas variáveis reais a valores reais por meio dos ostensivos algébrico e gráfico para estudar o domínio e a imagem destas funções e fazer as representações deste domínio e das curvas de nível. 
Neste livro, todas as noções introduzidas são acompanhadas de tarefas em que se exploram os níveis técnico, mobilizável e disponível, mas nas tarefas propostas aos estudantes, verifica-se que existe uma preocupação em articular os conhecimentos matemáticos com as outras ciências e os autores apontam a qual área do conhecimento a tarefa está associada.

Dessa forma, os autores desenvolvem um trabalho que, em função da contextualização proposta, exige um empenho maior dos estudantes, pois existem muitas tarefas em nível disponível para as quais o estudante deverá dispor de conhecimentos relacionados a outras áreas ou buscá-los.

Trata-se de uma obra que dá ao professor a possibilidade de fazer escolhas, mas que, como a anterior, exige que se trabalhem os três níveis de conhecimento tanto para a nova noção que está sendo introduzida, quanto para as noções esperadas como disponíveis para os estudantes do ensino superior.

\section{Análise da obra Cálculo de Stewart (2013)}

A noção de derivada parcial é introduzida pelo autor no volume 2 , o que já indica que se supõe que os estudantes disponham de conhecimento das noções de limite, derivada e integral, seus teoremas e propriedades associadas às funções de uma variável real a valores reais.

Mas o autor inicia esse volume propondo um teste de conhecimentos sobre noções básicas de álgebra, de geometria analítica no plano, funções polinomiais e trigonometria.

Além disso, o autor apresenta brevemente um estudo histórico sobre as áreas de polígonos inscritos em uma circunferência, o problema da tangente a uma curva, o limite de uma sequência e a soma de uma série.

Após introduzir as equações diferenciais, equações paramétricas e coordenadas polares, sequências e séries infinitas, vetores e geometria no espaço, funções vetoriais e curvas espaciais, derivadas e integrais de funções vetoriais é proposto um capítulo para o estudo das derivadas parciais.

Neste capítulo, o autor define função de duas variáveis reais por meio dos ostensivos: língua natural, numérico (tabela de valores), algébrico e gráfico (curva de nível), que o autor considera como diferentes pontos de vista. São propostos exemplos variados de determinação do domínio e imagem dessas funções e é indicado o uso do computador para visualizar as curvas de nível de diversas funções.

$\mathrm{Na}$ sequência, são introduzidas às noções de funções de três ou mais variáveis, superfície de nível, limite e continuidade. Todas essas noções são desenvolvidas por meio dos 
ostensivos algébrico e gráfico e o ostensivo numérico é utilizado para criar as imagens mentais necessárias à compreensão das noções em jogo.

A noção de derivada parcial é definida para as funções acima consideradas e após a introdução de derivada de segunda ordem, teorema de Clairaut, equações diferenciais parciais (equação de Laplace), plano tangente e aproximações lineares, diferenciais, regra da cadeia, diferenciação implícita, derivadas direcionais e vetor gradiente, plano tangente à superfície de nível e reta normal, máximos e mínimos locais e absolutos, pontos críticos e teste da segunda derivada, são considerados exemplos e propostas tarefas em que o nível de conhecimento esperados dos estudantes é o mobilizável e disponível, em particular, para os exemplos do domínio da Física, que exigem conhecimentos que extrapolam os desenvolvidos na Educação Básica.

Consideramos tratar-se de uma obra mais indicada aos estudantes dos cursos de Matemática e Engenharia, podendo ser utilizada nos cursos de Licenciatura em Matemática, todavia, nesse caso, exige um cuidado do professor, que deve considerar momentos para explicitar conceitos e noções de outras ciências, em particular, do domínio da Física.

\section{Considerações finais}

A avaliação do funcionamento institucional via livros didáticos deixa evidente que existe uma preocupação em ultrapassar as técnicas de derivação associadas à noção de derivada parcial.

A abordagem dessa noção proposta nos livros analisados ultrapassa o nível técnico das reproduções das mesmas tarefas, exigindo os níveis mobilizável e disponível, níveis estes que estão associados à articulação dos conhecimentos já existentes com os novos conhecimentos, supondo uma organização desses conhecimentos.

Além da utilização de diferentes ostensivos, é preciso estar atento às necessidades extramatemáticas para que os estudantes possam compreender as tarefas propostas que dependem de conhecimentos das outras ciências, mais especificamente, nas obras de Hoffmann e Bradley (2002) e Stewart (2013).

A análise das praxeologias associada ao estudo dos níveis de conhecimento esperados dos estudantes se mostra bastante eficaz, pois possibilita a identificação dos níveis de conhecimento esperados dos estudantes e possibilita assim uma melhor compreensão das necessidades dos mesmos, o que pode auxiliar professores a desenvolverem seus cursos a partir de um ponto de referência considerado como conhecido pelos estudantes, mas que, certamente, poderá ser negociado em função do maior ou menor grau de conhecimento demonstrado efetivamente por esses estudantes. 


\section{Referências}

CHEVALLARD, Y. Pour une aproche anthopologique du rapport au savoir. Dialogue 155, Paris, jan. 2015. Disponível em: http://www.gfen.asso.fr/images/documents/publications/dialogue/dial155_enligne_anthropo_rap_savo ir_chevallard.pdf $>$. Acesso em: 16 jun. 2017.

CHEVALLARD, Y. Organisations didactiques: 1. les cadres généraux. França, 1998. Disponível em: $<$ http://yves.chevallard.free.fr/spip/spip/article.php3?id_article=38 >. Acesso em: 16 jun. 2017.

CHEVALLARD, Y. Ostensifs et non-ostensifs dans l'activité mathématique. França, 1994. Disponível em: < http://yves.chevallard.free.fr/spip/spip/article.php3?id_article=125>. Acesso em: 16 jun. 2017.

CHEVALLARD, Y. Concepts fondamentaux de la didactique: perspectives apportées par une approche anthropologique. Recherches en didactique des mathématiques, Grenoble, vol.12, n. 1, p. 73-112, 1992.

DIAS, M.A. Les problèmes d'articulation entre points de vue cartésien et paramétrique dans l'enseignement de l'algèbre linéaire. 500 f. França, 1998. Disponível em: < https://tel.archivesouvertes.fr/tel-01252758 >. Acesso em: 16 jun. 2017.

DOUADY, R. Des apports de la didactique des mathématiques à l'enseignement. Repères - IREM, n. 15 , p. 37-61, 1992.

DOUADY, R. Jeux de cadres et dialectiques outil-objet dans l'enseignement des Mathématiques. Une réalisation dans tout le cursus primaire. $263 \mathrm{f}$. França, 1984. Disponível em: < https://tel.archives-ouvertes.fr/tel-01250665 >. Acesso em: 16 jun. 2017.

DUVAL, R. Sémiosis et pensée humaine. $1^{a}$ ed. Paris: Peter Lang, 1995.

GUIDORIZZI, H.L. Um curso de cálculo. 5ª ed. Rio de Janeiro: LTC Editora, 2004.

HOFFMANN, L.D. et BRADLEY, G.L. Cálculo : Um curso moderno e suas aplicações. $7^{\mathrm{a}}$ ed. Rio de Janeiro : LTC Editora, 2002.

ROBERT, A. Outils d'analyse des contenus mathématiques à enseigner au lycée à l'université. Recherches en didactique des Mathématiques, vol. 18, n. 2, p. 139-190, 1998.

ROBERT, A. Niveaux de conceptulisation et enseignement secondaire. In : DORIER, J.L. (Org.). L'enseignement del'algèbre linéaire en question. Grenoble : La Pensée Sauvage, 1997, p. 149-157.

ROGALSKI, M. Les changements de cadre dans La pratique des mathématiques et les jeux de cadres de Régine Douady. In:Équipe DIDIREM (Org..). Actes de la journée en hommage à Régine Douady. Paris: IREM Paris 7, 2001.

STEWART, J. Cálculo. $7^{\mathrm{a}}$ ed. São Paulo : Cengage Learning, 2013.

THRUSTON, W.P. Preuve et progress en mathématiques, Repères IREM, n. 21, p. 5-26, 1995.

Recebido em 23/03/2017 - Aceito em 31/10/2017 